\title{
Corps en ré-volution. Entretien avec le metteur en scène Mokhallad Rasem lors du colloque international « Re-Moving Apartheid »
}

Lichamen in re-volutie: in gesprek met regisseur Mokhallad Rasem tijdens het congres 'Re-moving Apartheid'

\section{Marieke Breyne et Sofie de Smet}

Traducteur : Emilie Syssau

\section{CpenEdition}

Journals

\section{Édition électronique}

URL : https://journals.openedition.org/temoigner/5692

DOI : 10.4000/temoigner.5692

ISSN : 2506-6390

\section{Éditeur :}

Éditions du Centre d'études et de documentation Mémoire d'Auschwitz, Éditions Kimé

Édition imprimée

Date de publication : 2 avril 2017

Pagination : 21-26

ISBN : 978-2-930953-00-7

ISSN : 2031-4183

Référence électronique

Marieke Breyne et Sofie de Smet, « Corps en ré-volution. Entretien avec le metteur en scène

Mokhallad Rasem lors du colloque international « Re-Moving Apartheid » , Témoigner. Entre histoire et mémoire [En ligne], 124 | 2017, mis en ligne le 30 novembre 2021, consulté le 01 décembre 2021. URL : http://journals.openedition.org/temoigner/5692 ; DOI : https://doi.org/10.4000/temoigner.5692 


\section{CORPS EN RÉ-VOLUTION}

\section{Entretien avec le metteur en scène Mokhallad Rasem lors du colloque international «Re-Moving Apartheid »}

THEATRE Les 28, 29 et 30 septembre 2016 s'est tenu au centre d'arts Vooruit à Gand le colloque international Re-Moving Apartheid, Postdramatic and Postnarrative Modes of Coping with Trauma (Détruire/re-muer l'apartheid, outils post-dramatiques et post-narratifs pour gérer le traumatisme). Ce forum de réflexion sur les vestiges traumatiques de l'apartheid, de la violence et de la discrimination en rapport avec l'art a été co-organisé par le centre de recherche S:PAM de l'Université de Gand, la KU Leuven et la School of Arts de Gand. Le groupe de recherche S:PAM et les co-organisateurs partenaires d'Afrique du Sud ont ainsi interrogé de façon critique le discours positiviste qui domine actuellement les études de traumatisme.

L 'intérêt dominant porté à la narration de l'expérience, remède à un traumatisme personnel et culturel, est ancré dans un univers de connaissance occidental. Dans diverses disciplines s'élèvent à présent des voix critiques qui remettent en question le caractère apparemment universel du traumatisme. L'intitulé du colloque, Re-Moving Apartheid, souligne cette intention : un traumatisme ne peut être simplement éliminé ou «removed», et il n'existe pas de procédure standard pour se remettre d'un traumatisme. Au contraire, ce colloque explore le processus complexe du mouvement continu - «re-moving »-en marge et au sein d'une expérience traumatique personnelle et culturelle. Un mouvement que l'on ne peut analyser de façon purement rationnelle. Le colloque a exploré la force motrice de l'art dans ce mouvement, d'une part à travers de nombreuses lectures, contributions universitaires, salons d'artistes, conférencesperformances, et d'autre part via un volet artistique mêlant ateliers, performances et exposition.

La performance Body Revolution s'inscrit dans le volet artistique. Ce spectacle de 2014 est signé par le metteur en scène et acteur Mokhallad Rasem, qui, après sa formation théâtrale à Bagdad, a fui la ville durant la guerre. Il habite en Belgique depuis 2005, et, depuis 2013, est dramaturge associé au Toneelhuis d'Anvers. Dans Body Revolution, il explore les réactions physiques à la violence et à la peur - les réactions d'un corps plongé au cœur de la violence, mais aussi mis à distance par la violence - par exemple celui de Rasem quand il a des nouvelles des membres de sa famille ou de ses amis qui vivent encore dans une région en guerre. Dans Body Revolution, Rasem transforme le fait de revivre des expériences de violence et de guerre en une installation intime où se succèdent les souvenirs.

En se concentrant sur cette rencontre incarnée, le spectacle sans paroles de Rasem rejoint remarquablement les questionnements du colloque international où il a été joué. Body Revolution confronte le corps numérique (les tweets quotidiens, les likes et les applications auxquelles nous nous sommes tant habitués) à la présence des corps physiques, propre au théâtre. Les acteurs et le public, parce qu'ils se trouvent dans une même pièce, partagent en outre cette incarnation, et expérimentent aussi une quête commune et incessante d'un corps nouveau, un corps qui se relève toujours de la douleur et de la destruction. Un corps qui veut continuer d'espérer. À l’issue de la représentation, une 


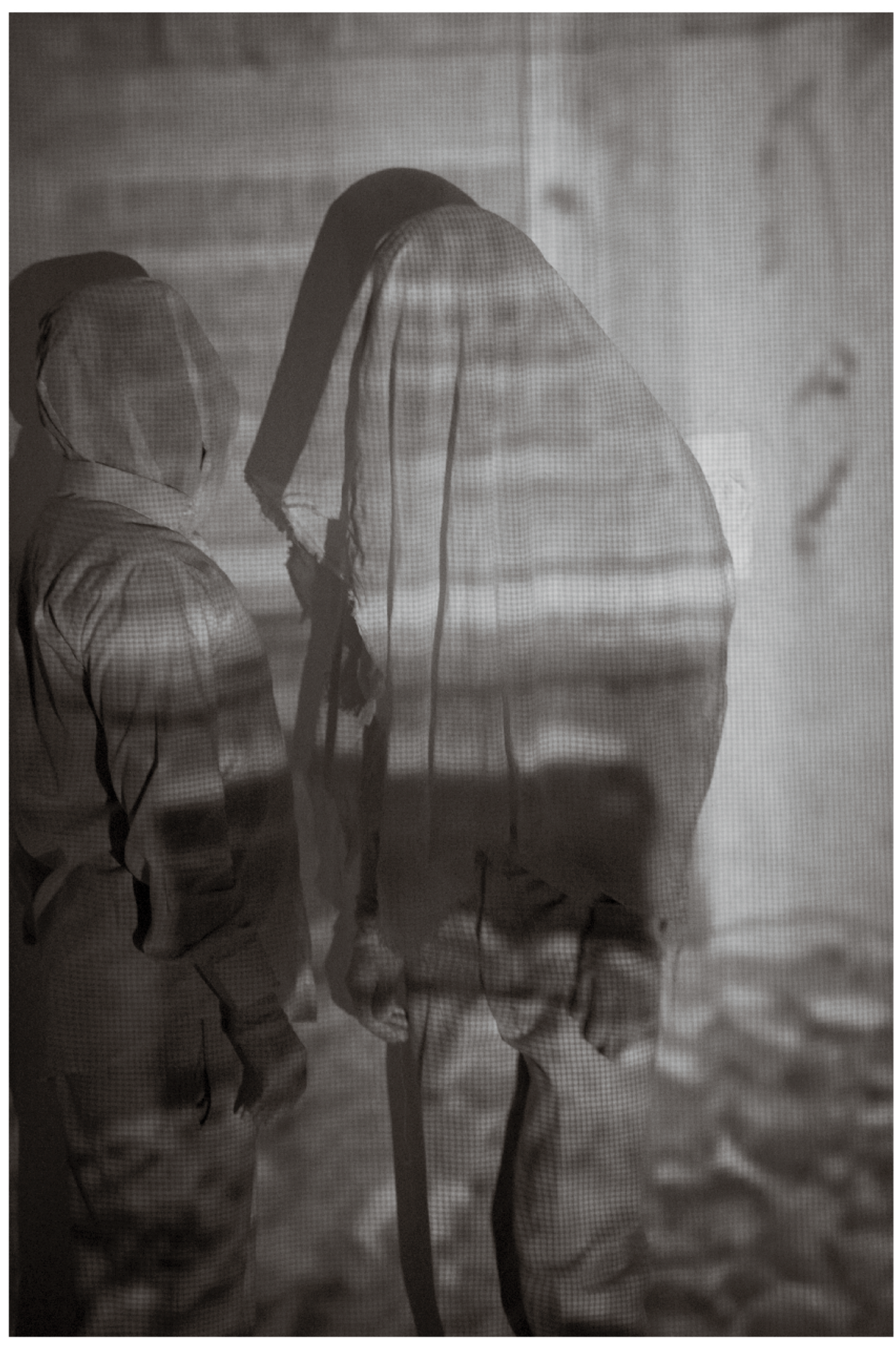

•. discussion entre le metteur en scène Rasem et le public s'est concentrée sur la force de cette rencontre.

LE CORPS NUMÉRIQUE

Dans Body Revolution, des photos de destruction et de violence sont projetées sur les corps des acteur vêtus de blanc. Ceux-ci semblent ainsi apparaître rédisparaitre dans la fumée ou dans des batimen reduits en ruines, conne sils avaient disparu tout en restant présents. Par lenplor de cet écran humain, les inages de guerre - connues via les réseaux sociaux ment par les acteurs, mais aussi par le public.

Mokhallad Rasem : En réalité, la scène est un iPad. déflent exactenc Des images défilent, exactenent comme les images défilent sur Facebo Sur Facebos, on peut facilom de bang et de guer. Sur l'́cran si on peut facilement balayer ces inases de lectran ou si on veut les empecher de sinsinuer dans ses pentant qu'Irakien. je ne veux pas lo voir. C'est une forme d'autoprotection : je ne peux pos porter toutes d autoprotection : je ne peux pas porter toutes ces tion je cherche unquilibre entre cette autoprotection et l'impossibilité de véritable chette autoprotection

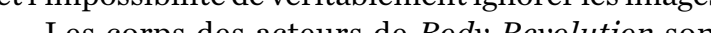
Les corps des acteurs de Body Revolution son épartis dans lespace par le jeu avecles 'eftet de las. riques, ils semblent se disperser sous leffet de la vioun pied devient une brare d'arbre se passe lors d'une explosion: On ne sait plus où se passe lors dune explosion: On ne sait plus où se pysique, mis sau physque, maíe par la vionn est fragmentee par la violen? Lom question est alors : il? Le flot d'imacos ? Body Revolution représente donce que la violent. Body Revolution représente don ce ce la violence et la guerre infligent réllent aux hommes, et veut dont ils abordent ces images, et y restent insens à distancer

En même temps, j'essaie de ne pas simplement illustrer l'horreur de la guerre qui écartèle les corps et

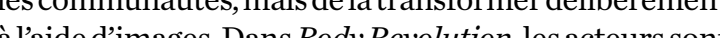
alacés, en live ou dans des vidéos, dans les images pro- jetées des ruines. La chorégraphie des acteurs met en jouvement les images de destruction. En recourant à la stratification des images, j'essaie de créer quelque chose de nouveau à partir de la destruction et de souligner l'aspect visuel de la guerre.

Je compare parfois les corps de Body Revolution à une bôte remplie de Kleenex. Les mouchoirs en à une boite remplie de Kleenex. Les mouchoirs en papît sont les âmes du spectacle. On en sort un de

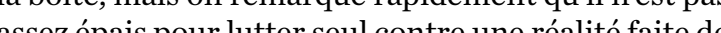
assez épais pour lutter seul contre une réalite faite de punker dise varte lles solco elles sont confrontées à la souffrance. Elles perdent en direction et donc en force. Dans le spectacle, on voit ce téte, é plus à egare par la soutfrance physique, ne parvient

LE CORPS PARTAGÉ

La représentation terminée, le silence perdure. Linncartion partagée de drames historiques souligne peut-être dans ce silno respo de responsabilté. En Belgique, la violence guerière nous a Qu Body Revoluton a tes'ausendre prente? Body Revolution a été su sera presenté?

Le spectateur etablit par alleurs rapidement un lien biographique entre le materiau historique et les acteurs sur scene Cetre a distinction entre représentation et présentation de la douleur.

Les réactions du public sont très variées. En Roumanie, nous avons par exemple eu des réaction Roumanie, nous avons par exenple eu des réactions étonnamment violentes. La Roumanie n'est pas en à la dureté de la vie qui lui était présentée. En Iran à lá dureté de la vie qui lui était présentée. En Iran et sûr au sûr au Rwanda. A Kigali, nous avons joué au Genocide de 2000 pes, comble, le jour même de la citrode 2000 places, comble, lejour même de la commémosilence. Au contraire, le public était excité silence. Au contraire, le public était excité. A Anvers,
nous avons effectué une semaine d'ateliers avec un 
• groupe d'étudiants ; ils n’ont pas osé applaudir à la fin du spectacle. Ils trouvaient vraiment trop étrange 'applaudir, et sont donc restés assis en silence. C'e

Il y a une grande différence entre un public qui reconnaît des images de son vécu et un public qui ne fait qu'observer ces images a distance. Chaque lieu pré propres et qui influencent la perception du propres et qui infuencét la perception du public. Ici en Europe,le spectacle Guerre mondiale.

Nous ne choisissions pas délibérément de joue dans des lieux où la population est actuellement ou éré des lieux où la population est actuellement ou a êx qui nous demandent d'interver. En eux qui nous demandent dintervenir. En novembre, ous ín en Tun une, là s'est immolé par le feu. cetinte quar es flant, les flammes et la place. Cetait mon souhait d'aller en Téactions et des conversations jue nous y arons.

Selon moi, personne ne doit se sentir coupable. Nous sommes tous victimes de ce qui se passe dans le monde. La violence est devenue très universelle, elle ne se limite pas au monde arabe. La peur est partout.

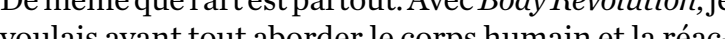
tiouls avant tout aborder le corps humain et la réachistoire d'homs à la violence. Body Revolution est une histoire dhommes ayant vecu la guerre et une explodans cet espace partagé. Eshan (acteur) vient pur Sons cet espace partagé. Eshan (acteur) vient d Iran. con que celui de Bassim (acteur) ou que le mien. Sur scène, férents corps. Il n'y est question ni de notre histo ferrents corps. Il ny est question ni de notre histoire, l'effet de la violence, mais du corps qui souffre sous

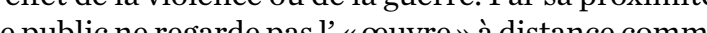
le public ne regarde pas $\mathrm{I}$ " œuvre» a distance comm ience Aujourd'hui, ceétait la toute première fois que je jouais dans ce spectacle. Avant le début, je pensais Jaijuste à suivre la chorégraphie, et à faire les geste correspondants.» Mais en jouant, et seulement la, j’a combien je suis submergé dans l'instant, combien j’ai conscience des choses dans l'instant. Eshan m’a raconté qu'il entend une sirène d'alarme à divers moments du spectacle Un britiméc spectacle. Un bruit inaginé, ou absorbé, qui le replonge sûr pour échapper aux raids aériens.

LE CORPS NEUF

Au début du spectacle, nous regardons durant de longues minutes la projection en noir et blanc du tableau Les horreurs de la guerre du peintre français Paul-Émile Boutigny. Une présence saute aux yeux dans le tableau les personnages féminins. La blancheur de leurs corps nus contraste avec les personnages sombres : des hommes à cheval, en armure, au milieu d'un champ de bataille. Les femmes ne sont pas représentées sous des trait doiques. Elles semblent noyées par des sentiments $d$

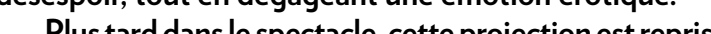

Plus tard dans le spectacle, cette projection est reprise en couleurs. Les trois acteurs masculins apparaissent

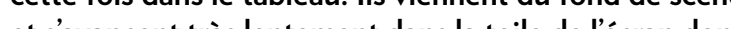
et savancent tres le ils senveloppent. Le spectateur attentif remarque que Nous taches de sang, ni corps inanimés, ni victimes.

J'ai délibérément pris pour point de départ ce monde abîmé. Le résultat d'une guerre est un monde brisé. La guerre en soi est déjà très dure, mais ce qui lui fait suite est enso est jeja tres dure, mais ce quilui guerre ent encore plus dur. Les conséquences de la guerre sont plus dures que la guerre même. On perd da plachs chers monde, son âme. Pour moi, Eton perd sartou dans le monde, son âme. Pour moi, cela parle surtout des mères. Un pays est conme une mère qui donne vie à de nouveaux êtres. Le corps féminin symbos êtres. Un pays enguere est comoune unse mìre des êtres. Un pays en guerre est comme une mère qui me ler une femme. A la guerre, les hommes meurent et les J'associe à l couleur bont les principales victimes. J'associe a la couleur blanche une page vierge, une vie nouvelle. En Irak, on enveloppe les nouveau-nés dans des draps blancs. Les morts aussi. La vie débute et se tersine en blanc. Les draps blancs dans lesquels breuses sours s'avancent symbolisent aussi les nommoi ceste trasition de couche Le spectacle est pour toile, jusquau cœur des souvenirs.
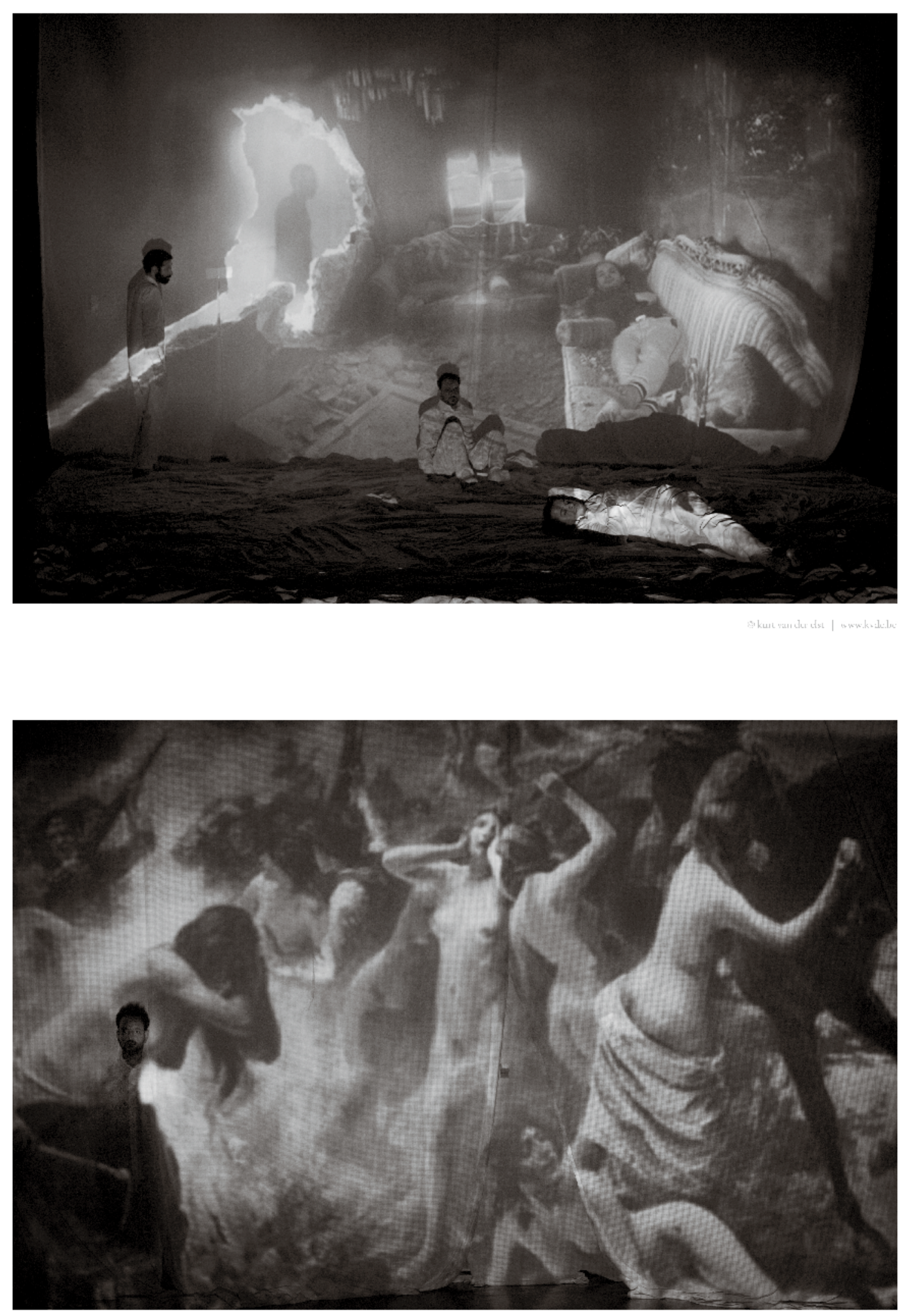
—. Après les expériences de violence guerrière, il est bien sûr impossible de se retrouver face à une page totalement blanche - une tabula rasa. Éléments anciens et nouveaux se mêlent, d'où une stratification. J'essaie de transformer dans ce matériau ce qui subsiste après la guerre. Je transforme la douleur, le chagrin, la ruine en quelque chose qui puisse redonner vie. Je ne veux pas arrêter de bouger. S'arrêter, c'est mourir. Mais comment relire la réalité quand tout est brisé ? C'est aussi une question d'espoir. J'aimerais faire renaître quelque chose, même à partir de la peur ou de la mort. L'imagination est un instrument puissant, où l'on est aussi chez soi.

Mais que signifie précisément l'espoir ? L'espoir signifie que l'on parvient à voir de la lumière même dans l'obscurité. J'ai l'espoir que personne ne souffre dans ce monde, qu'il n'y ait ni violence ni peur. Que tout soit raccommodé. À l'instar des toiles du spectacle : à chaque représentation, ils se déchirent un peu plus, mais nous les réparons toujours. Nous utilisons encore les draps blancs de la première, et nous les emmenons partout. Ils rentrent dans une valise, et avec cette valise nous faisons le tour du monde.

Nous avons également joué Body Revolution en Iran. Étant Irakien, je n'y étais encore jamais allé la dictature l'a en effet interdit pendant 35 ans. Nous avons grandi avec le message clair que l'Iran était notre ennemi. Pouvoir, après tout ce temps, jouer enfin ce spectacle en Iran, avec des collègues iraniens, et découvrir ce pays et ses habitants, était un symbole d'espoir. L'espoir que la guerre peut vraiment cesser.

\section{RÉ-VOLUTION}

Notons que Body Revolution contient le même suffixe «re-» que le titre du colloque, Re-moving Apartheid. Rasem s'est inspiré de la Révolution arabe, mais l'univers sans paroles de sa création puise surtout dans le sens étymologique du mot révolution : «re-volvere» ou re-tourner, revenir. Le titre du colloque renvoie aussi à cette étymologie. Comme nous l'avons indiqué dans l'introduction, le terme «re-moving» critique ironiquement le discours dominant sur les traumatismes, selon lequel un traumatisme doit être guéri, soulagé ou éliminé. Parler de souvenirs de violence implique au contraire des mouvements complexes et perpétuels - «re-moving» ou «re-muer» - dans toutes les directions. Le corps dessiné par la violence et la perte ne peut revenir simplement au corps qu'il était avant. Le corps en fuite dans Body Revolution continue à re-muer indéfiniment dans des draps blancs.

Le lendemain du spectacle, le dramaturge Mandisi Sindo est intervenu pour parler de son travail dans les townships de Kayelitsha, Gugulethu et Delft en Afrique du Sud. Dans sa présentation, il nous a indiqué une autre interprétation du titre du colloque « Re-Moving Apartheid », en déplaçant l'attention de l'expérience traumatisante à son origine, à savoir la violence même qui ne doit être ni « removed» ni « détruite» :

Let us look for another word rather than 'removing'. Think about it this way: if you 'remove' that chair, you are planning to put it down somewhere else, but if you 'destroy' that chair, you will throw it in the dust bin and move on. That is what needs to be done with apartheid. It needs to be destroyed, not just removed. The question remains: how?

(Cherchons un autre mot que «removing». Réfléchissons ainsi : « retirer » une chaise implique que l’on prévoit de la mettre ailleurs, mais la «détruire», c'est la jeter à la poubelle et passer à autre chose. C'est ce qu'il faut faire avec l'apartheid. Le détruire, pas simplement le retirer. La question demeure : comment?)

La discussion consécutive à la représentation de Body Revolution réunissait les différents corps sur scène : le corps numérique, le corps partagé et le corps neuf. Sindo attire l'attention sur un quatrième corps, auquel tous les autres sont liés : le corps perpétrant un acte de violence. Pour mettre un terme à la création de nouveaux corps traumatisés, il faut mettre un terme à la violence et à la guerre.

Rasem s'interroge lui aussi : «Pourquoi l'homme doit-il chaque fois témoigner et dire : "nous devons arrêter de faire la guerre", alors que les guerres perdurent ?» Selon Rasem, la force de l'art réside précisément dans sa capacité à transformer le regard du spectateur et à lui faire prendre conscience de son rôle dans la société: «L'art peut sérieusement remettre en question notre façon de regarder la violence, de la décrire, la dessiner et la jouer.» |

Marieke Breyne \& Sofie de Smet (UGent) Traduit du néerlandais par Emilie Syssau 